\title{
Draft Genome Resources for Plant-Beneficial Fungi Clonostachys rosea Strains ACM941 and 88-710
}

\author{
Zerihun A. Demissie, Kelly A. Robinson, and Michele C. Loewen ${ }^{\dagger}$ \\ Aquatic and Crop Resource Development, National Research Council of Canada, Ottawa, Canada
}

Funding

This work was funded by the National Research Council of Canada grant number 014211.

\section{Keywords}

biocontrol, fungus-plant interactions, genomics, metabolomics, transcriptomics

Clonostachys rosea, a member of the family Bionectriaceae in the order Hypocreales (Schroers 2001), is an aggressive necrotrophic mycoparasitic filamentous fungus. Based on this property, a few strains isolated from diverse habitats have been developed as biocontrol agents against an array of plant pathogens, including Botrytis cinerea, Sclerotinia sclerotiorum, and Fusarium species (Lysøe et al. 2017; Sutton et al. 1997; Zhang et al. 2008). Strains ACM941 and 88-710, respectively, are patented in North America as biocontrol agents against $F$. graminearum (the causal agent of Fusarium head blight disease) and for plant growth promotion (Stewart and Brown 2012; Xue 2002). However, these properties are not mutually exclusive to each strain. Strain $88-710$ is superior in promoting plant growth and development but can also impart some disease resistance (Sutton et al. 1997), while ACM941 is superior in its mycoparasitic properties. The molecular and biochemical basis of these properties, however, remains poorly understood.

The genome of $C$. rosea CBS125111 has been sequenced and annotated as part of the 1,000 Fungal Genome Project initiative, while genomes of strains IK726, YKD0085, and 67-1 were reported by different groups (Karlsson et al. 2015; Liu et al. 2016; Sun et al. 2015a). While the IK726 strain has shown antagonism against Alternaria spp. (Karlsson et al. 2015; Lysøe et al. 2017), Bipolaris sorokiniana, Fusarium culmorum, Pythium spp., and Tilletia tritici (Karlsson et al. 2015; Lysøe et al. 2017), strain 67-1 is best known to antagonize S. sclerotiorum (Sun et al. 2015b). In addition to functional and geographic overlap, C. rosea strains ACM941 and 88-710 are genetically closely related (Demissie et al. 2019). Therefore, we hypothesized that comparative genomics along with omics profiling could be used to identify the genetic material (both regulatory and structural) and corresponding metabolites

\footnotetext{
${ }^{\dagger}$ Corresponding author: M. C. Loewen; michele.loewen@nrc.ca
}

The author(s) declare no conflict of interest.

Accepted for publication 24 December 2020.

๑) 2021 NRC Canada 
Table 1. Genome assembly statistics of Clonostachys rosea strains

\begin{tabular}{lccc} 
Genome statistics & $\mathbf{I K 7 2 6}^{\mathbf{a}}$ & $\mathbf{8 8 - 7 1 0}$ & ACM941 \\
Genome fraction (\%) against IK726 & 99.997 & 91.916 & 93.619 \\
\# contigs & 595 & 593 & 822 \\
Largest contig & $3,780,011$ & 373,352 & $3,733,882$ \\
Total length & $58,326,963$ & $55,501,686$ & $56,858,285$ \\
Number of contigs $(\geq 1,000 \mathrm{bp})$ & 595 & 583 & 814 \\
Total length $(\geq 1,000 \mathrm{bp})$ & $58,326,963$ & $55,501,686$ & $56,858,285$ \\
$\mathrm{~N}_{50}$ & 794,244 & 824,329 & 805,045 \\
$\mathrm{~N}_{75}$ & 365,347 & 425,882 & 366,156 \\
$\mathrm{~L}_{50}$ & 24 & 22 & 23 \\
$\mathrm{~L}_{75}$ & 51 & 45 & 49 \\
$\mathrm{GC}(\%)$ & 50.08 & 50.24 & 50.12 \\
$\mathrm{~N}$ per $100 \mathrm{~kb}$ & $1,613.65$ & $1,570.03$ & 954.6 \\
\hline
\end{tabular}

a From Karlsson et al. (2015).

conferring these differential properties. Here, we report the sequencing, assembly, and annotation of strains 88-710 and ACM941 genome.

Clonostachys rosea ACM941 (American Type Culture Collection ATCC 74447) and 88710 (active ingredient of EndoFine, Adjuvants Plus, Canada) were grown at $25^{\circ} \mathrm{C}$ for 10 to 12 days and were maintained on potato dextrose agar plates at $4^{\circ} \mathrm{C}$. Freeze-dried mycelium was ground to a fine powder and genomic DNA was extracted using EZNA Fungal DNA mini kit following the manufacturer guidelines (Omega Bio-tek). Genomic DNA libraries (550 bp insert size) were prepared using TruSeq Nano DNA library prep kit, followed by sequencing on Illumina MiSeq 2500 platform (30x coverage) according to the manufacturer guidelines (Illumina) at NRC-Saskatoon DNA sequencing facility (Saskatoon, Canada).

Trimmomatic trimmed paired-end $C$. rosea ACM941 and 88-710 reads were mapped using default settings of the "map reads to contigs" tool within the de novo sequencing module of CLC-Genomics Workbench software (Qiagen), using C. rosea IK726 genome (Karlsson et al. 2015) as reference, with "update contig" and "save unmapped reads" boxes checked. This was followed by de novo assembly of unmapped reads (approximately $5 \%$ of total reads), using the de novo sequencing module of CLC-Genomics Workbench. SPAdes assembly pipelines based on the De Bruijn Graph was also used for both de novo and hybrid assembly using IK726 PacBio reads (Short Read Archive [SRA] ERX2621092 [Broberg et al. 2018]) for comparison (Bankevich et al. 2012). The genomic sequences were annotated using AUGSTA (Keller et al. 2011) and the Maker genome annotation pipeline (Campbell et al. 2014) tools from the Galaxy server. Genome quality was assessed with Quast using IK726 genome as a reference (Gurevich et al. 2013).

A total of 18,262,482 (average length $=298.6 \mathrm{bp}$ ) and 16,141,674 (average length $=$ $298.3 \mathrm{bp}$ ) reads were obtained for $C$. rosea ACM941 and 88-710 genomes, respectively. The raw genomic reads were processed into 18,259,417 (average length $=292.3 \mathrm{bp}$ ) and $16,138,454$ (average length $=291.5 \mathrm{bp}$ ) high-quality reads, respectively, using Trimmomatic software in the Galaxy platform (Bolger et al. 2014). As shown in Table 1, ACM941 reads were assembled into a total genome size of $56.9 \mathrm{Mb}\left(N_{50}=805,045 \mathrm{bp}, N_{75}=366,156 \mathrm{bp}\right.$, and GC content of $50.1 \%$ ) composed of 822 scaffolds, with its largest scaffold containing $3.73 \mathrm{Mb}$. On the other hand, strain 88-710 reads were assembled into 593 scaffolds for a total length of $55.5 \mathrm{Mb}\left(N_{50}=824,329 \mathrm{bp}, N_{75}=425,882 \mathrm{bp}\right.$, with \% GC content $\left.=50.24\right)$ and largest contig size of $3.73 \mathrm{Mb}$ (Table 1). These values are within the expected range of previous $C$. rosea genome assembly reports (Karlsson et al. 2015; Liu et al. 2016; Sun et al. 2015a). The $N_{50}$ values of SPAdes assembly outputs were lower (data not shown), thus excluded from further analysis.

ACM941 scaffolds were annotated to 17,585 genes, of which 527 were annotated from de novo assembled contigs. Blast2go functional annotation identified homologous sequences for 10,825 ACM941 genes in the SwissProt database and only 925 (5.26\%) of the transcripts failed to find a hit in the nonredundant (nr) database. Similarly, 88-710 genome was annotated to 17,188 genes, of which 404 were annotated from de novo assembled contigs. Only $897(5.22 \%)$ of the $88-710$ transcripts failed to find a hit in the nr database. Pairwise alignment of these transcripts, using the megablast module of the National Center 
for Biotechnology Information BLAST+ blastn tool, showed that $>96 \%$ of the ACM941 encoded genome is homologous to that of 88-710, with almost all transcripts sharing $>90 \%$ nucleotide identity. On the other hand, approximately $98.3 \%$ of the $88-710$ genome encoded transcripts are homologous to that of ACM941 with $>90 \%$ nucleotide identity. This further implies that the biological activity differences between these strains arises either from less than $4 \%$ of their respective encoded genomes, differentially expressed homologous genes, or both. Only 73.8 and $74.4 \%$ of the putative transcripts of ACM941 and 88-710, respectively, were found to have homologous transcripts in the reference genome of $C$. rosea IK726. The seemingly low transcript homology level to the reference genome compared with their overall genome similarity (Table 1 ) is likely due to the low number $(14,268)$ of putative transcripts derived from the reference genome (Karlsson et al. 2015).

Secondary metabolites are the leading candidates to mediating plant growth promotion and fungicidal properties of beneficial microbes (Vinale et al. 2009). AntiSMASH analysis of the draft genomes revealed that strains ACM941 and 88-710 contain the same number of gene clusters anchored by terpene (10) and polyketide (29) synthases. However, ACM941 and 88-710 genomes contain 28 and 27 nonribosomal peptide synthetase-anchored gene clusters and 11 and 10 gene clusters containing more than one type of secondary metabolism anchor gene, respectively (data not shown). Similarly, comparable numbers of ACM941 and 88-710 transcripts (1,274 and 1,240, respectively) with gene ontology (GO) terms associated with transmembrane transport were identified. On the other hand, 6,061 and 5,503 ACM941 transcripts were mapped to GO terms with oxidoreductase and ion binding activities, respectively, while only 4,770 and 3,203 strain $88-710$ transcripts had similar GO terms, respectively (data not shown). In conclusion we sequenced, assembled, and annotated the first draft genomes of two beneficial $C$. rosea strains with potential impact to sustainable agricultural practices in the future. These genomic sequences will be considered in future studies to understand their respective beneficial properties.

The draft whole-genome sequence reads and assembly are deposited in GenBank under accession number JACYFL000000000 and JACYFM000000000 for strain ACM941 and 88-710, respectively, under BioProject PRJNA656487.

\section{Acknowledgments}

We acknowledge A. Xue (Agriculture and Agri-Food Canada, Ottawa, Canada) and W. G. Brown (Adjuvant Plus Inc., Kingsville, Canada) for providing C. rosea strains ACM941 and 88-710. The SRA accession numbers associated with these assemblies are SRR12475922 and SRR12475921. This manuscript represents NRCC communication number 58259.

\section{Literature Cited}

Bankevich, A., Nurk, S., Antipov, D., Gurevich, A. A., Dvorkin, M., Kulikov, A. S., Lesin, V. M., Nikolenko, S. I., Pham, S., Prjibelski, A. D., Pyshkin, A. V., Sirotkin, A. V., Vyahhi, N., Tesler, G., Alekseyev, M. A., and Pevzner, P. A. 2012. SPAdes: A new genome assembly algorithm and its applications to single-cell sequencing. J. Comput. Biol. 19:455-477.

Bolger, A. M., Lohse, M., and Usadel, B. 2014. Trimmomatic: A flexible trimmer for Illumina sequence data. Bioinformatics 30:2114-2120.

Broberg, M., Dubey, M., Sun, M. H., Ihrmark, K., Schroers, H. J., Li, S. D., Jensen, D. F., Brandström Durling, M., and Karlsson, M. 2018. Out in the cold: Identification of genomic regions associated with cold tolerance in the biocontrol fungus Clonostachys rosea through genome-wide association mapping. Front. Microbiol. 9:2844.

Campbell, M. S., Holt, C., Moore, B., and Yandell, M. 2014. Genome annotation and curation using MAKER and MAKER-P. Curr. Prot. Bioinform. 48: 4.11.1-4.11.39.

Demissie, Z. A., Brown, W. G., and Loewen, M. C. 2019. A universally primedpolymerase chain reaction (UP-PCR) marker to discriminate Clonostachys rosea ACM941 from related strains. J. Fungi (Basel) 5:39.

Gurevich, A., Saveliev, V., Vyahhi, N., and Tesler, G. 2013. QUAST: Quality assessment tool for genome assemblies. Bioinformatics 29:1072-1075.

Karlsson, M., Durling, M. B., Choi, J., Kosawang, C., Lackner, G., Tzelepis, G. D., Nygren, K., Dubey, M. K., Kamou, N., Levasseur, A., Zapparata, A., Wang, J.,
Amby, D. B., Jensen, B., Sarrocco, S., Panteris, E., Lagopodi, A. L., Pöggeler, S., Vannacci, G., Collinge, D. B., Hoffmeister, D., Henrissat, B., Lee, Y.-H., and Jensen, D. F. 2015. Insights on the evolution of mycoparasitism from the genome of Clonostachys rosea. Genome Biol. Evol. 7:465-480.

Keller, O., Kollmar, M., Stanke, M., and Waack, S. 2011. A novel hybrid gene prediction method employing protein multiple sequence alignments. Bioinformatics 27:757-763.

Liu, S., Chang, Y., Hu, X., Gong, X., Di, Y., Dong, J., and Hao, X. 2016. Draft genome sequence of fungus Clonostachys rosea strain YKD0085. Genome Announc. 4: e00538-16.

Lysøe, E., Dees, M. W., and Brurberg, M. B. 2017. A three-way transcriptomic interaction study of a biocontrol agent (Clonostachys rosea), a fungal pathogen (Helminthosporium solani), and a potato host (Solanum tuberosum). Mol. PlantMicrobe Interact. 30:646-655.

Schroers, H.-J. 2001. A monograph of Bionectria (Ascomycota, Hypocreales, Bionectriaceae) and its Clonostachys anamorphs. Stud. Mycol. 46:1-214.

Stewart, J. F., and Brown, W. G. November 2012. Production and use of endophytes as novel inoculants for promoting enhanced plant vigor, health, growth, yield reducing environmental stress and for reducing dependency on chemical pesticides for pest control. European Patent EP2001821A4.

Sun, Z.-B., Sun, M.-H., and Li, S.-D. 2015a. Draft genome sequence of mycoparasite Clonostachys rosea strain 67-1. Genome Announc. 3:e00546-15. 
Sun, Z.-B., Sun, M.-H., and Li, S.-D. 2015b. Identification of mycoparasitism-related genes in Clonostachys rosea 67-1 active against Sclerotinia sclerotiorum. Sci. Rep. 5:18169.

Sutton, J. C., Li, D.-W., Peng, G., Yu, H., Zhang, P., and Valdebenito-Sanhueza, R. M. 1997. Gliocladium roseum a versatile adversary of Botrytis cinerea in crops. Plant Dis. 81:316-328.

Vinale, F., Flematti, G., Sivasithamparam, K., Lorito, M., Marra, R., Skelton, B. W., and Ghisalberti, E. L. 2009. Harzianic acid, an antifungal and plant growth promoting metabolite from Trichoderma harzianum. J. Nat. Prod. 72 : 2032-2035.

Xue, A. G. December 2002. Gliocladium roseum strains useful for the control of fungal pathogens in plants. U.S. Patent US6495133B1.

Zhang, L., Yang, J., Niu, Q., Zhao, X., Ye, F., Liang, L., and Zhang, K.-Q. 2008. Investigation on the infection mechanism of the fungus Clonostachys rosea against nematodes using the green fluorescent protein. Appl. Microbiol. Biotechnol. 78:983-990. 